\title{
Microscopy101
}

\section{Stereoscopic Photography in Transmitted Light Microscopy}

\section{Robert Sturm}

University of Salzburg, Department of Physics and Biophysics, Hellbrunnerstr. 34, A-5020 Salzburg, Austria

Robert.Sturm@stud.sbg.ac.at

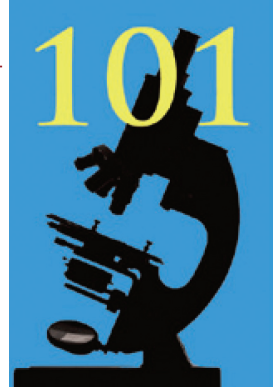

Abstract: The present contribution describes two techniques for the efficient generation of scientifically useable 3D images. The first method is the production of stereo pairs by photographing a microscopic object from two slightly different perspectives, achieved by rotating the sample slide by $5-10^{\circ}$. The second method involves the generation of an image stack, where the focus plane is stepped through a transparent object. At each position of the focus plane a separate image is recorded. Stereo pairs or stacked images are subsequently converted to anaglyphs using specific computer software. Applicability of the described techniques is demonstrated with several examples.

\section{Introduction}

Scientific photography is frequently employed to produce realistic undistorted depictions of the spatial extent of an object. The spatial dimensioning of an object can be surmised only partially from a single image; whereas, threedimensional (3D) information can be stored with the help of two semi-images (now called a stereo pair) that portray the object from two slightly different perspectives. If these semi-images are separately directed to the eyes, so that the left eye exclusively perceives the left image and the right eye is confronted with the right image, the primary visual cortex initiates a fusion of the pictures using its specific "spatial sense." Viewing of these two-dimensional (2D) images together thus evokes the identical reaction in the brain as would emerge from a glance at the object itself $[1,2]$.

Stereoscopic viewing. The stereoscopic principle was developed over many years starting in the 17 th century and climaxing in the second half of the 19th century, when photography advanced to a routine method. In the latter period, the classical stereoscopic technique using two semi-images placed side by side was enhanced by alternative presentation methods. For example, the well-known anaglyphic method uses the filter colors red and green, red and cyan, or red and blue for the pair of images. By application of bicolored glasses with an identical combination of filter colors, each eye perceives the corresponding image, resulting in the generation of a spatial impression [3-7].

Both the classical stereoscopic technique and the anaglyphic method have been used in scientific photography from the beginning of the 20th century. During recent decades, stereophotography received more attention in microscopy because a comprehensive description of small objects demands it [8-10].

Stereo microscopy. In scanning electron microscopy (SEM), 3D photography is facilitated by tilting the sample holder.
This method has been used to produce millions of $3 \mathrm{D}$ images of small objects ranging in size from $50 \mathrm{~nm}$ to $5 \mathrm{~mm}[7,11]$. In light microscopy, however, this optical procedure is frequently stretched to its limits, because viewing of embedded objects from two different perspectives is possible only to a limited extent [4-7]. Transmitted light microscopy is commonly used for the study of transparent objects between $1 \mu \mathrm{m}$ and $100 \mu \mathrm{m}$ in size. The present article describes two simple methods for the production of high-quality stereo images using the transmitted light microscope along with suggestions for computer software enabling the fast preparation of anaglyphs of stereo pairs.

\section{Material and Methods}

Typical items examined in stereo by transmitted light include microfossils (for example, radiolarians, diatoms, etc.) and microorganisms (for example, bacteria, animal and plant cells, etc.). As outlined in detail in previous publications [4-7], these objects are usually embedded in a highly viscous substance with high light refraction (for example, Canada Balsam, $\mathrm{n}=1.53$ ) to accentuate their contours under the microscope.

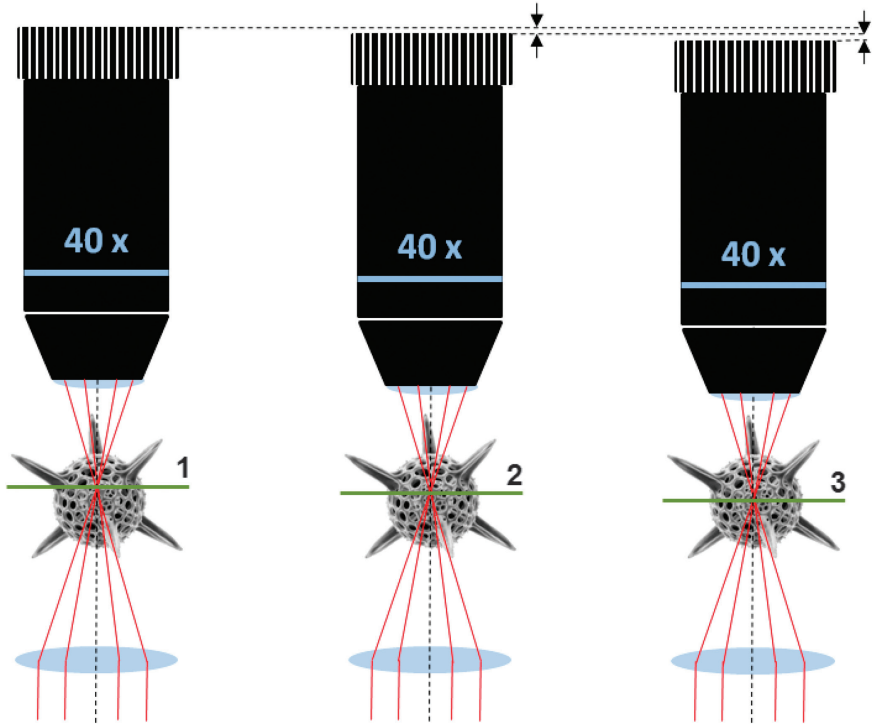

Figure 1: Generation of stereo images with the transmitted light microscope. A transparent object (e.g., a radiolarian) is recorded at different focus planes (1-3), where the distance between two focus planes (dashed lines at the top) is a few micrometers. 


\section{The moment "I think"}

becomes "I know".

This is the moment we work for.

I/INNOVATION

MADE BY ZEISS
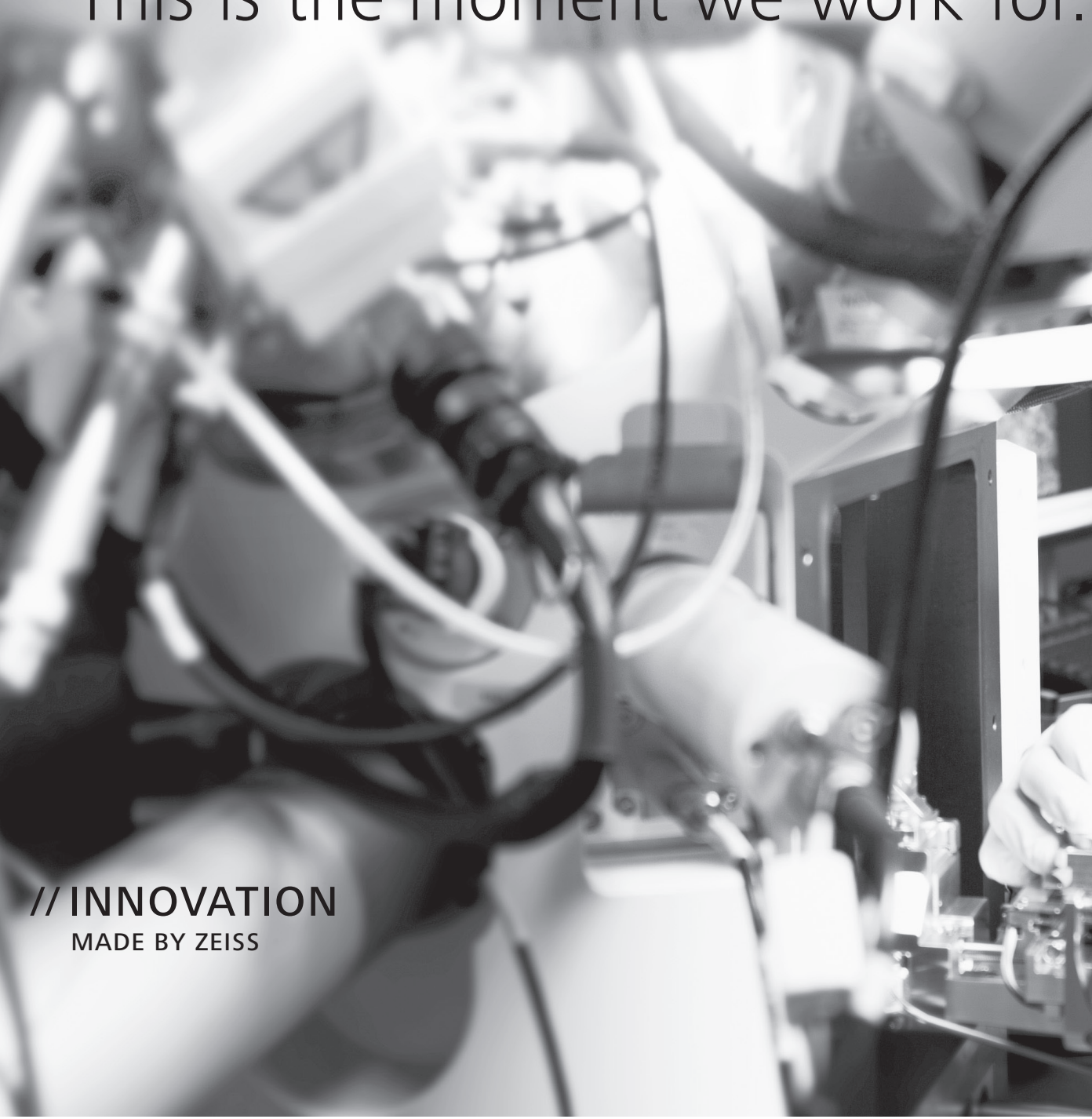


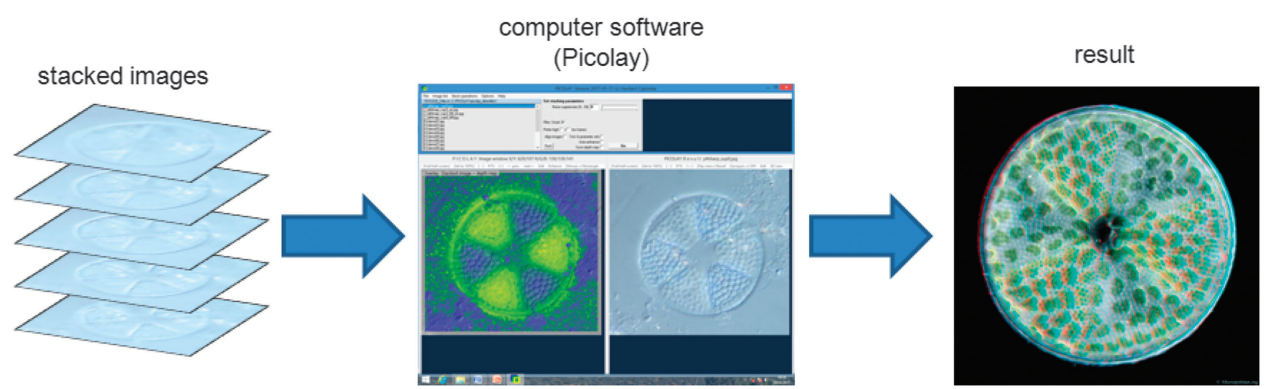

Figure 2: Digital processing of stacked raw images generated according to the method illustrated in Figure 1. Fusion of the images and subsequent creation of an anaglyphic photograph may be carried out using the program Picolay [8].

Collection of data for subsequent construction of 3D (stereo) images can be conducted in two principal ways.

Stereo-pair method. The first method records images of an object from two perspectives with a small angle in between. For this purpose, the object is recorded in its "normal" position by placing the slide with the item to be studied on the sample stage. For the second image, the slide is slightly rotated about the light axis of the microscope (rotation angle: $5-10^{\circ}$ ), and the same object is recorded a second time. For these two images, an identical setup (magnification, light intensity, etc.) must be used. This method for 3D imaging is most useful for the case of small items exhibiting spherical or close-to-spherical geometry.

Image stack method. The second method for 3D imaging is useful for anisometric objects. Here the selected object is recorded in the same position, but the focus plane is moved step-wise through the object creating an image stack (Figure 1). Photographs with a number of different focus planes within a small transparent object require use of rather high objective magnifications (for example, $40 \times, \mathrm{NA} \sim 0.65$ ) to limit the depth of field captured at each focus plane. In principle, distances between the focus planes depend on the size of the object, but

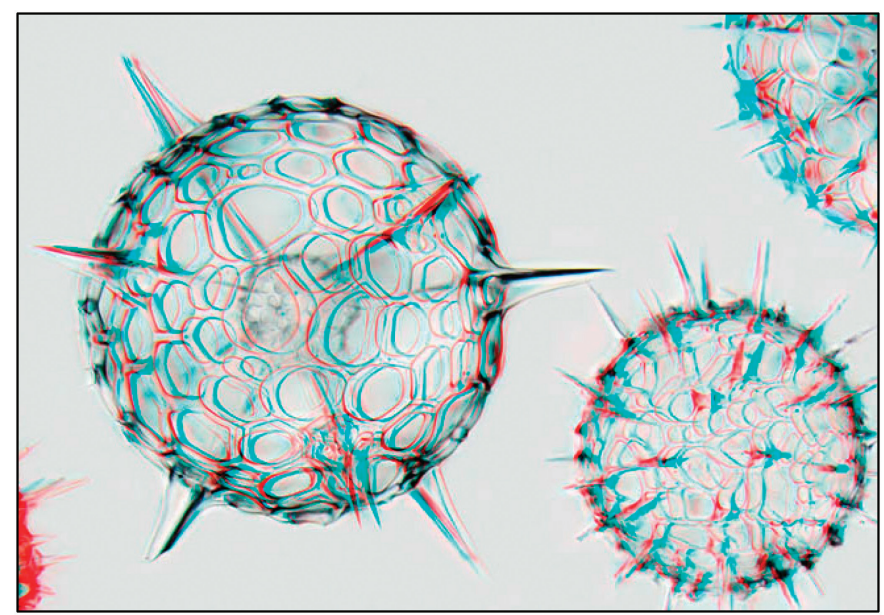

Figure 3: Anaglyphic photograph of globular Radiolaria with typical spines that provide buoyancy of these unicellular microorganisms. By using low magnification (4x, NA 0.10), generation of stereo pairs was possible by method 1. Microscope: NIKON Eclipse, $40 \times$, NA $~ 0.65$. Image width $=200 \mu \mathrm{m}$. Semi-images are copyright of and used with permission of Wim van Egmond, micropolitan.org. they should be on the order of a few micrometers. For stereo imaging, it is highly recommended to make a stack containing 10 to 20 images.

Production of 3D images. For the generation of anaglyphic images viewable with special red-blue glasses, stereo pairs (method 1) or stacked images (method 2) must be processed digitally using appropriate computer software. A stereo pair of images may be transformed into an anaglyphic photograph via various dedicated computer programs (for example, Anaglyph Maker; https://anaglyph-maker.de.softonic. $\mathrm{com} /$ ) or with Adobe Photoshop ${ }^{\mathrm{TM}}$ [3-7]. In the latter case, tutorials explaining the steps of anaglyph generation are available online (for example, http://designinstruct.com/photography/3danaglyph-photoshop/). For processing of stacked images into an anaglyphic photograph, the freely available computer code Picolay (www.picolay.de) [8] is a powerful digital tool. This program commonly enables the processing of high numbers of raw pictures and offers numerous opportunities for digital image manipulation (Figure 2).

\section{Results}

Radiolaria. In paleontology, 3D images of microfossils can be used for detailed descriptions of shell structures and for a more secure determination of single species. Radiolarians, especially those with globular shells, have become favorite items for $3 \mathrm{D}$ imaging in recent years. This is because they can be easily photographed by applying the methods of stereo pairs or stacked images noted above (Figure 3). The fossil shells of these microorganisms commonly vary between several tens and several hundred micrometers in size and are therefore

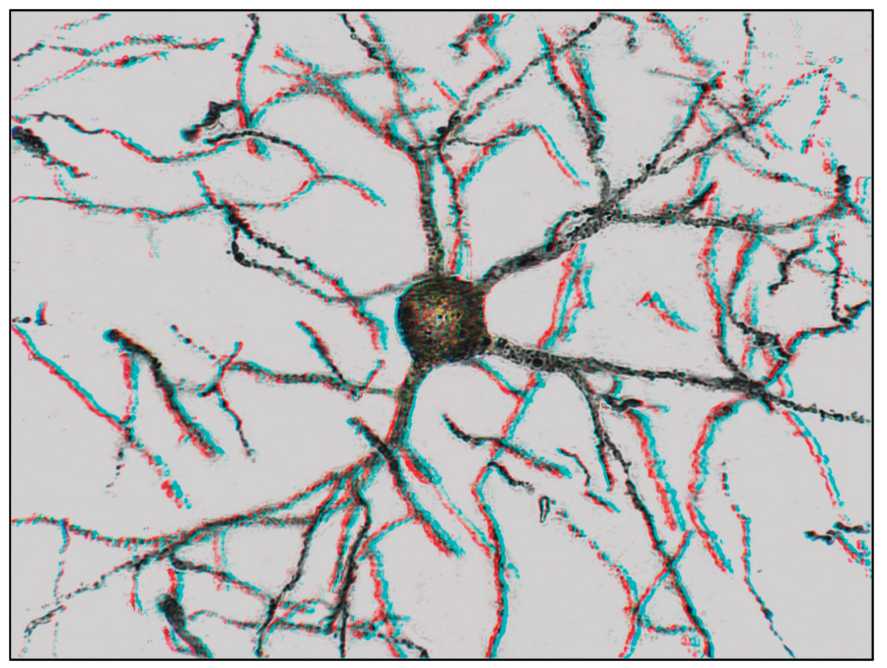

Figure 4: Detailed view of a rat neuron and associated dendritic processes stained with silver. The 3D image was dendritic from an image stack containing 10 pictures. Microscope: ZEISS Primotech, $40 \times$, NA $\sim 0.65$. Image width $=200 \mu \mathrm{m}$. Semi-images are copyright of and used with permission of Eberhard Raap, micropolitan.org. 


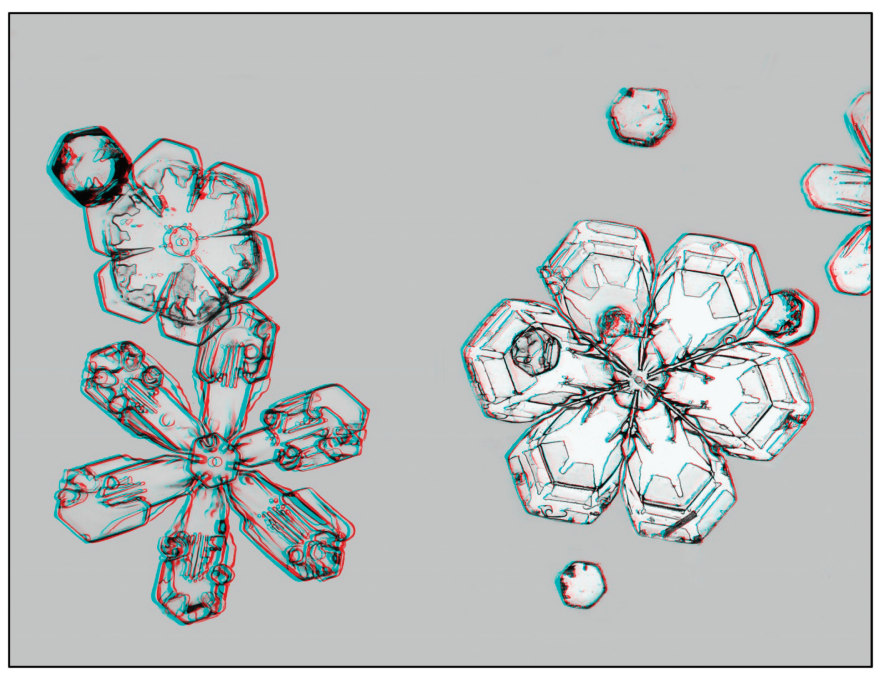

Figure 5: Stereophotograph of snow crystals developing different hexagonal geometries. Because of the flatness of these objects, production of raw images with the stacking method illustrated in Figures 1 and 2 is highly recommended. Microscope: ZEISS Primotech, 10x, NA 0.25. Image width $=2 \mathrm{~mm}$. Semi-images are copyright of and used with permission of Eberhard Raap, micropolitan.org.

viewable with modest magnifications. The preparation of rocks containing such fossils (radiolarites), however, turns out to be rather time-consuming because the shells have to be corroded out of the stony material with the help of hydrogen peroxide.

Neurons. Another application for stereo photomicrography is in biological tissue studies. Figure 4 shows the $3 \mathrm{D}$ view of the brain of a rat, underlining the significance of this technique for microanatomical investigations. Here the production of stacked images with individual focus planes is highly recommended because of the limited thickness $(30 \mu \mathrm{m})$ of the histological section. For an optimization of the spatial resolution, distances between images in the stack should not exceed $2 \mu \mathrm{m}$. Single dendritic cells such as the one in the center of the anaglyphic photograph have a diameter of about $20 \mu \mathrm{m}$ and therefore have to be documented at higher magnifications. With the help of 3D images, courses of single dendrites and axons can be traced more easily, and the 3D arrangement of single nerve cells within the brain can be measured.
Snowflakes. Microscopic documentation of snow crystals with their multitude of shapes has developed into a particular eye-catcher (Figure 5), although preparation of such objects involves numerous complications and therefore requires a high degree of experience [7,8]. Snow crystals are distinguished from the other items described here by their platelet-like geometries, where one dimension $(z)$ is significantly reduced with respect to the other two $(x, y)$. This circumstance requires the production of raw images according to the stacking method. Three-dimensional imaging of the crystals measuring several hundred micrometers in size may be used for a more comprehensive exploration of the internal structure or the comparison of thicknesses developed in variably shaped objects.

\section{Conclusion}

We describe here two simple methods for the production of stereo images in transmitted light microscopy. Whilst the first technique, conventional stereo pairs, is chiefly restricted to isometric items of intermediate size $(100 \mu \mathrm{m}$ to $5 \mathrm{~mm})$, the second technique, image stacks, is appropriate for small objects of any shape and size down to a few micrometers in extent. From the 3D micrographs exhibited here, it may be concluded that stereoscopic imaging represents a microscopy method whose scientific potential is not exhausted. In some research disciplines, the method is well-established, while in others it has not yet been explored.

\section{References}

[1] C Wheatstone, Transactions of the Royal Society of London 128 (1838) 371-94.

[2] D Brewster, The stereoscope: Its history, theory, and construction. J. Murray, London, 1856.

[3] R Sturm, Mikrokosmos 97(2) (2008) 75-80.

[4] R Sturm, Deposits Magazine 18 (2009) 10-13.

[5] R Sturm, Mikrokosmos 98(6) (2009) 331-36.

[6] R Sturm, Deposits Magazine 26 (2011) 12-15.

[7] R Sturm, Mikroskopie 3(2) (2016) 86-100.

[8] E Raap and H Cypionka, Mikrokosmos 100(3) (2011) 140-44.

[9] R Sturm, Biologie in unserer Zeit 45(1) (2015) 52-55.

[10] R Sturm, Naturwissenschaftliche Rundschau 70(4) (2017) $500-06$.

[11] JI Goldstein et al., Scanning Electron Microscopy and $X$-ray Microanalysis. Springer, New York, 2003.

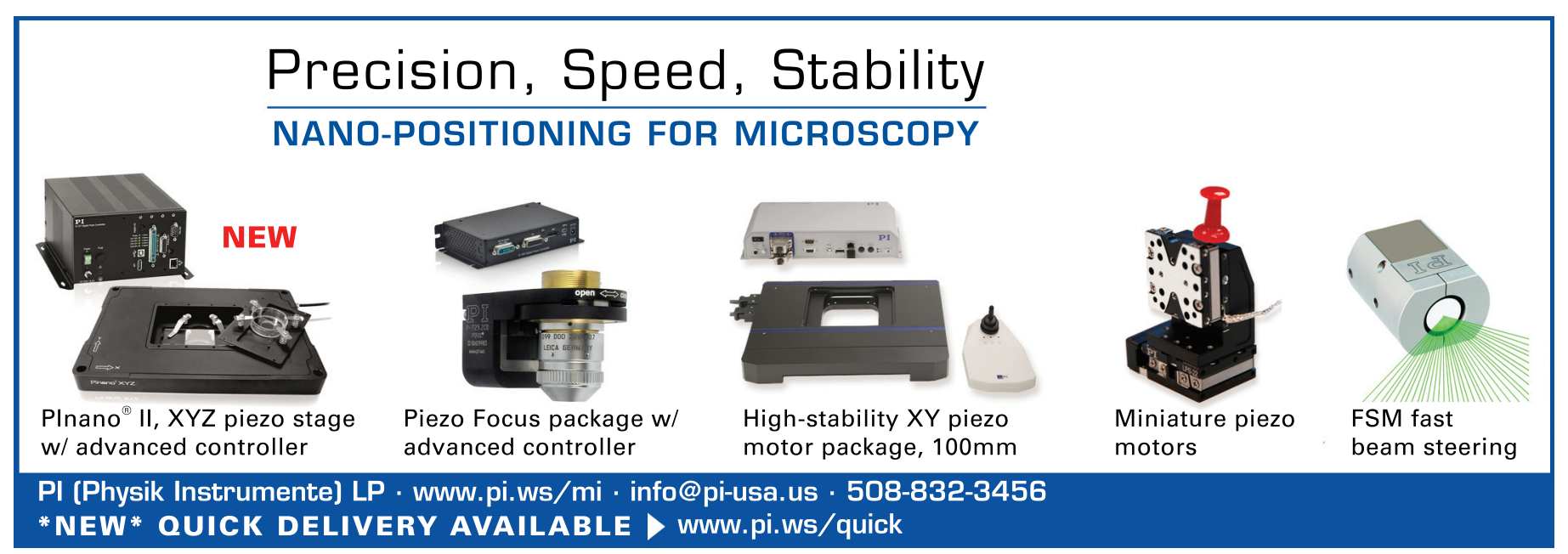

